\title{
PREVENTION AND TREATMENT OF ULCERS AND DEFORMITIES IN LEPROSY
}

Manfrleis J. Oberdörffer and Douglas R. Collikr.

The object of this paper is to present our observations and opinions on the subject of ulcers and deformities in leprosy. The importance of the subject is well known to those working in leper colonies and need not be stressed. In order to limit the scope of this paper, it appears necessary to give a short pathogenic classification of the symptoms concerned and to select that group which we intend to discuss.

Ulcers in leprosy may be of various origins :-

I. Granulomas of lepromatous or tuberculoid structure in the skin may ulcerate. All these ulcers, including typical lepra lazarina (rich in bacilli) are a direct consequence of infection with mycob. leprae, and apart from ordinary dressings require the treatment of leprosy itself. They will be omitted from this discussion.

2. Ulcers may be due to injury of anaesthetic parts of the the limbs. While we believe that minor injuries are one of the important factors in the development of all ulcers, we do not in this 
paper wish to deal with greater injuries, burns, etc., which require well known, conventional methods of treatment.

3. Ulcerating " trophic" skin lesions may develop in the course of pachydermia, general atrophy of the skin or neurotrophic bullae. Their localisation does not correspond with the type of ulcer with which we are now concerned. Their treatment is essentially the same.

4. Trophic or static ulcers form the group of ulcers the treatment of which we intend to describe. In addition we wish to draw attention to certain deformities of bones and muscular disturbances in limbs, their treatment and prevention.

Before analysing " trophic" deformities and ulcers, we wish to exclude from discussion all those cases in which there is actual formation of lepromatous granuloma in the bones. Such inflammatory foci similar to syphilitic and other bacterial affections of the bone-marrow may of course lead to deformities and mutilations. They are however by no means frequent in leprosy and in our experience are only found in advanced lepromatous cases. Their treatment is the specific treatment of leprosy and need not be discussed here.

We have therefore limited this paper to those symptoms in leprosy which are considered a secondary consequence of lepromatous or tuberculoid infiltration of the large nerve-trunks in the limbs. The well-known symptoms are deformities, mutilations, muscular paralysis and ulcers.

We feel that the term " trophic disturbance" as describing the underlying condition of ulcers and deformities is a very vague one. What is meant is that a disturbance of nerves results in secondary symptoms in the area supplied by the nerve affected. It is presumed, but not proved, that apart from motor and sensory fibres there exist so-called trophic nerve-fibres which regulate the nutrition and the metabolism of the area supplied. We do not deny that such fibres might possibly exist, but we are inclined to base our explanation of observations largely on better established facts. Clinical and pathological analysis of the symptoms concerned reveals the following :-

I. In all of them there is evidence of damage of one or several large nerve trunks, such as the ulnaris, the medianus, the radialis, the peroneus, the musculo-cutaneous, the tibialis posterior. The lesion producing this damage is different in each type of leprosy. (Grieco, Takino, Jermakova, Ermakova, Chatterji). In lepromatous cases there is a more diffuse infiltration with Virchow cells which is not very vigorous and does not destroy many of the nerve-fibres. In marked tuberculoid cases 
there is vigorous, well-limited tissue-reaction, sometimes with abscess-formation. In this type the mechanical pressure of the inflammatory focus results in rapid destruction of nerve-fibres. The tissue reaction in the simple neural cases is only a minor degree of what in advanced types is called tuberculoid. Clinically, soft thickening or induration due to scar-formation is found in palpation of the nerves, particularly in certain well-known predilected parts such as the ulnaris above the elbow, etc.

2. The most constant symptom of nerve-involvement is anasthesia to slight touch, pain or temperature. Indeed we do not remember any case of secondary deformity in leprosy in which there was no symptom of sensory disturbance in the area concerned.

3. In all cases with deformities we find various degrees of muscular paralysis, from slight reduction of muscular strength and visible wasting to complete ulnar, peroneal or facial paralysis. We wish to add here-and this holds good for the distribution of acro-anaesthesia as well-that in distinct lepromatous cases anaesthesia and muscular wasting are not extensive and usually without the marked limitation seen in neural-tuberculoid cases. Here, and in the simple neural case which is a minor stage of the same, we find that muscular wasting and anaesthesia is frequently restricted to one small group of muscles and to one well-marked area of sensory disturbance. Moreover, unilateral deformities are frequently seen in neural-tuberculoid, but scarcely ever in purely lepromatous cases. The picture however becomes more complicated in those cases which, while in their preliminary stage they are neural-tuberculoid, later turn lepromatous. As will be seen later, this difference of intensity of fibre-destroying tissue-reaction in the two clinical types of leprosy has an important bearing on the pathogenesis and treatment of deformities.

4. In lepromatous cases we frequently find a diffuse, noninflammatory oedema of hands or feet which may often persist for a long time and is often, if not regularly, followed by mutilation. (Plate I). We have never seen this acro-oedema in neural cases. The significance of this symptom will be discussed later when we come to describe the pathogenesis of deformities.

5. Deformities and mutilations are present in various degrees. We find simple contractures of fingers and toes. Later, shortening of hands or feet, while finally mutilation in the form of retraction takes place. In nerve leprosy these mutilations are frequently of a different shape to those in lepromatous leprosy. We have recently studied the Röntgenology of a number of deformed and mutilated cases and agree with the findings of Leloir, Deycke, Hirschberg and Biehler, Businco, Wayson and Garland, Nonne, 
Hayashi, Walter, Murdoch and Hutter, Wooley and Ross, Harbitz and Lee, Jame, Jakob and Jude. We found that the essential change is decalcification, and in a few cases we have found lepromatous osteomyelitis and periostitis, as did Beitzge and Hirschberg and Biehler. As noted above, we consider this event to be a rare one. The reason for decalcification has been explained in various ways. There is no agreement in the biochemical findings so far, and the rarity of specific lepromatous infiltration makes one believe that the decalcification must be a secondary process connected with the involvement of the nerves. We exclude here periostitis leprosa and the rare primary affections of joints in leprosy. Our own findings, which have been recently published in detail (Oberdörffer-Collier), give strong evidence for the following idea of pathogenesis of these deformities. We found that in neural-tuberculoid cases the decalcification is most marked in those bones which receive insertions from paralysed or semi-paralysed muscles, while in lepromatous cases decalcification and mutilation is more diffuse and more marked in the small bones at the ends of the limbs which carry insertions of small muscles. From these observations we deduced the following theory.

Decalcification is the direct consequence of complete or partial musculary paralysis. The bones receive their blood-supply not only from the central nutrient artery, but also from the periosteal vessels which enter the bone through the insertion of the muscles. It is known (Häupl) that the process of calcification and decalcification in bones is regulated by the regular intermittent change of hyperaemia and anaemia. This process can be disturbed by the blocking or constriction of the nutritional artery. Such an event leads to the group of diseases called Kienboeck's Disease, or aseptic necrosis. In leprosy we have never seen it. The leprotic affection of the sympathetic nerves which regulate the blcod supply through the nutritional artery is not common. Much more does the blood-supply of the periosteal vessels underlie interferences by leprosy. It is well known that active exercise leads to intensive calcification in those bones the circulation of which is supplied by the muscle in training. Leitner has recently described.observations which tend to show that in deformed limbs of lepers blood-vessels are generally dilated. This confirms our idea that semi-paralysed or fully paralysed muscles produce a stasis in the periosteal blood-supply of bones, and that the result is decalcification, particularly of those bones which receive muscular insertions from muscles enervated by damaged nerves in leprosy. We do not think that Harbitz's idea of the trophoneurotic origin of deformities in leprosy can be upheld in its full application. Nor do we consider osteoarticular periostitis 
(Karaseff, Murdoch and Hutter, Grainger) to be of primary nature in the development of mutilations. Decalcification is in our opinion essentially the consequence of muscular paralysis or semi-paralysis. One may answer then that similar deformities should be expected in cases of infantile paralysis or mechanical destruction of nerves. We think that the particular localised atrophy to a degree of disappearance of parts of the bones is due to the incomplete destruction of nerve-fibres in the different degrees or stages of leprotic involvement of nerves. It is natural that parts of the bones whose muscle insertions are supplied with unparalysed muscular fibres will remain intact, while in others the complete disappearance is due to the constant passive hyperaemia which is increased by functional activity in the unparalysed muscle groups in the vicinity. Details should be read in our original paper on the subject. We think however that this is not the full story. Decalcification of the bones does not occur besides after muscular inactivity in the area adjoining inflammatory or oedematous foci (Sudeck's Atrophy). We have stated above that in lepromatous cases we frequently observe an acro-oedema of hands or feet. We are inclined to believe that such acro-oedema may give rise to increased absorption of bones where a mild degree of inactivity has already prepared the path. This observation would explain the difference of degree and the difference of localisation in mutilations of the neural-tuberculoid and the lepromatous type. It may be added that in later stages secondary infections from penetrating wounds may lead to periostitic and calcified infiltrations. These, in our opinion, are entirely of secondary character except the rare cases mentioned above in which there is specific lepromatous periostitis.

Following these ideas and thus stressing the importance of muscular semi-paralysis secondary to nerve-lesions in leprosy, we find no difficulty in confirming the views of Muir et al. concerning the pathogenesis of trophic ulcers in leprosy. These ulcers, which are mostly confined to the sole of the foot, originate in the following way. The semi-paralysed muscles of the sole of the foot become thinner and offer less elastic resistance to the bones of the heel, of the big toe, or of the sesamoid bones or of partly necrotic phalanges. These pierce the skin, or produce such local pressure on the skin as to interfere with the blood-supply of the area concerned. This area of thin ischaemic skin will sooner or later give way to local injuries and the ulcer is formed. Secondary infection later may produce sequestration of bones or far-reaching destruction of subcutaneous tissue. The essential pathogenetic condition in the development of ulcers however is semi-paralysis of muscular and elastic tissue resulting in abnormal static pressure 
and finally in ulcer-formation. In this instance we see no reason to give undue importance to " trophic " disturbances.

As we intend to include another subject in our therapeutical notes, we wish to mention that facial paralysis is of essentially the same nature as muscular paralysis in the limbs, i.e. it is semiparalysis rather than a complete one.

We differentiate the following methods of attack in prevention and treatment of secondary deformities and ulcers in leprosy.

( I) Treatment of leprosy by specific methods is the primary object and need not be discussed here. Furthermore it is not within the scope of this paper to deal with general treatment as far as management of diet and conduct and treatment of predisposing diseases is concerned.

(2) Treatment of deformities and ulcers by intravenous injection of Mercurochrome, Synthol soufré (Tisseuil) and other disinfectants has so far not found general acknowledgment.

(3) The one point which should be considered and treated first in these conditions is the localised infection of the large nervetrunks. A vast number of drugs has been recommended for the subjective relief of nerve-pain in leprosy. So far, Ephedrine gives satisfactory results, though by no means in all cases. We consider the results obtained by De la Plaza, Vegas and Gomez, by De Moroes and Chopra by injection of crotalus toxin as a palliative and probably deplethoric application. The essential aim of therapy in the conditions concerned is, besides subjective relief of pain, the diminution of vigour of the inflammatory reaction in the nerve in order to prevent secondary destruction of nerve-fibres by oedematous pressure and the subsequent development of deformities and ulcers. Several methods are being employed to produce such a diminution of inflammatory reaction. Cochrane and Raj and others have recommended injection of alcohol along the nerve. Gupta tried cloretone in olive-oil, and others inject hynocarpus oil along the nerve. We are inclined to believe that in all these methods there is no specific factor involved, but that there merely occurs a transference of inflammatory reaction from the nerve to the surrounding tissue. Diathering is, according to Dow, of no value in acute nerve-reaction, though very beneficial afterwards. We think that in leper colonies where it is financially possible the use of a vitamin B.I preparation like Betaxin (Keil) should be given a trial. Though we do not think that vitamin B.I is a specific therapy for leprosy, the deplethoral action of this drug, particularly on nerve-tissue, might be employed. At present the best method of relieving oedematous pressure in nerve-reaction appears to us to be nerve-decapsulation. This has been recommencled and carried out by Chatterji, Gass and others. We are 
aware of Dow's sceptical attitude with regard to late results of such decapsulation. Decapsulation may even be harmful, if a too vigorous scar-formation follow the acute stage. We have therefore restricted our activities in this field of late to simple longitudinal splitting of the nerve-sheath, and so far the results have been very satisfactory. Indeed we have made this method the standard treatment of acute nerve-reaction in this leper colony. The restriction of scar-formation by this method to limited areas of nerve-sheath does, in our experience, prevent the fixation of surrounding tissue in a tough ring around the whole nerve, which so frequently occurs after decapsulation.

(4) Deformities without ulceration have so far found only limited interest in the treatment of leprosy. Mobilisation by active and passive exercise has been recommended by Dow, Denny, McIlhenny and Muir. We find however that while such measures as applied to already existing deformities may and do produce beneficial results, too little attention is being given to the preventive power of active exercise in the case of lepers who are still free from deformity. Following our theory on the development of deformities as a consequence of semi-paralysis of muscles following nerve-involvement, we consider it possible, and indeed probable, that active exercise of the particular muscle-groups concerned will prevent the development of deformities in general. We have therefore introduced for every leper here daily exercises as follows :-

The purpose of the exercises is to strengthen and train the small muscles of the hands and feet, namely the lumbricales, the interossei, the adductors and abductors of the thumb, and the corresponding muscles of the feet, the interossei, the flexor, abductor and adductor hallucis. Similarly there are exercises planned to train the facial muscles and the orbicularis oculi. We have used drums and other percussion instruments as a means of keeping time and promoting uniformity.

I. Vigorous opening and closing of the fist, i.e. alternate flexion and extension of the fingers combined with extension and flexion of the arms by a forward movement.

2. Standing on one foot, the other is raised forward and the foot rotated at the ankle. Repeated with the other foot.

3. Rotation of the thumbs. With the hand held forward the thumbs are rotated in as large an arc as possible. The two thumbs may be exercised together or one after the other.

4. With arms extended forward at shoulder height, the first finger of one hand is grasped with the other hand and forcibly extended with a backward bending movement. This is repeated for each finger of both hands. 
5. With the palms of the hands together the fingers are interlocked. Then the palms are spread apart and the fingers extended against each other as the hands are slowly raised as far as possible above the head, then brought down to chest level and as low as possible in front.

6. Bending at the waist, the outstretched hands touching the ground just in front of the toes, without bending the knees. This of course is a general exercise, not appertaining to the treatment of this particular disease.

7. Flexion of the toes. Standing on one foot the other is slightly advanced with the heel on the ground and the toes raised. They are then alternately flexed and extended in as large an arc as possible.

8. Deep breathing. With the hands on the hips the lungs are alternately expanded and contracted by deep breathing. This again is a general exercise.

9. Another general exercise. Standing on the toes, hands on hips, the body is lowered as far as possible by bending the knees while keeping the back straight.

Io. Facial movements. The mouth is opened widely and closed alternately. Then the mouth is moved laterally from one side to the other.

II. The eyes are alternately opened widely and closed. The movements are exaggerated so as to give the fullest motion possible to the eyelids.

Each of the above movements is repeated about ten times, the exercises occupying about half an hour daily.

Judging from the influence on existing deformities, we find that 70 per cent. of our N.3 cases (cases with deformities) have been considerably improved by the exercises. In the case of facial paralysis we add active massage of the eyelids and find that in a great number of cases this condition also shows marked improvement.

Unfortunately we cannot claim priority for this prevention and treatment of deformities, but are obliged to give full credit to the unknown artist who in the IIth century sculptured the basrelief in the Bayon at Angkor-Thom in Indo-China. Oberdörffer found that amongst these bas-reliefs there is one depicting a leprous king whose claw-hands and deformities are treated by active exercise.

The underlying idea of the preventive exercise is to produce increased calcification by increasing the activity of those muscles which are most likely to become semi-paralysed by nerveinvolvement in leprosy. This is particularly important in those lepromatous cases with acro-oedema in which the oedema forms a second and very potent cause of decalcification. 
We think that such exercises, by removing the predisposing cause of ulceration does at the same time in a great number of cases prevent it.

(5) Once ulcers have developed, we are concerned with a quite different problem, and many methods have been devised to deal with it. Various antiseptics and dyes have been recommended by Berny, Floreani and others. In our experience there is, so far, no drug which has found the approval of all leprologists. It goes without saying that in those cases where necrotic septic bone is present amputation or sequestrectomy affords the best results, but even then results are not always satisfactory. Periarterial sympathectomy (Black) has not found many followers. Other surgical methods with quite promising results are the free excision of the edges of an ulcer and skin transplantation. Other authors have reported on the beneficial action of .periulceral injection with chaulmoogra preparations. Though we do not doubt the beneficial results recorded, we fail to see how a drug which is supposed to act on the leprosy bacillus should have specific action on a secondary tropho-mechanic lesion. We think that the effect is only an unspecific stimulation of the tissue surrounding the ulcer. In the following we propose a new treatment of ulcers, but we certainly do not want to discredit other methods previously recommended. Indeed we think that our method would form a suitable combination with all other previous ones. We have described above what we consider is the pathogenetic process of ulcer formation. The theory that semiparalysis of muscles and static disturbances produced thereby are of paramount importance in the production of ulcers leads inevitably to the conclusion that static relief of pressure is the first condition to be fulfilled. We try to do so by padding the sole of the foot in such a way that the weight of the body no longer rests on the ulcer. These pads are made of canvas filled with rice-husk or other suitable material; they can easily be applied and fastened with a tape at each of the four corners. This method has been recommended before, but its importance has apparently been overlooked. In the local treatment of the ulcer we are very conservative. A weekly change of dressing, on which occasion the foot is well bathed in antiseptic fluid, is considered sufficient. The dressing is done with a thick application of the following ointment :

\begin{tabular}{|c|c|c|c|c|c|}
\hline urochrome & $\ldots$ & $\cdots$ & $\ldots$ & $\ldots$ & $2 \mathrm{oz}$ \\
\hline Honey & $\ldots$ & $\ldots$ & $\ldots$ & $\ldots$ & 8 \\
\hline Cod Liver Oil & $\ldots$ & $\ldots$ & $\ldots$ & $\ldots$ & 8 \\
\hline Zinc Oxide Powder & $\ldots$ & $\ldots$ & $\ldots$ & $\ldots$ & 4 \\
\hline Bismuth Subnitrate & $\ldots$ & $\ldots$ & $\ldots$ & $\ldots$ & 2 \\
\hline Vaseline & $\ldots$ & $\cdots$ & $\ldots$ & $\ldots$ & I 2 \\
\hline
\end{tabular}


Mercurochrome 2 per cent. scrves as disinfectant; honey is rich in ferments and stimulates scar-formation; cod-liver-oil has been found beneficial in other ulcerating conditions; zinc oxide serves as an absorbent; bismuth subnitricum as a mild astringent; and vaseline as a suitable carrier. The results of this treatment of combined padding and ointment are seen in the photographs. So far all the ulcers here have reacted very beneficially. The padding enables the leper to walk about and to get sufficient general exercise, while the ointment appears to have an unspecific beneficial effect. The experiments with this procedure were carried out without applying any other. We believe that combination with other surgical methods would give still better results.

In addition to our methods of prevention and treatment of ulcers and deformities in leprosy, we want to stress the importance of destroying by caustics or other methods reservoir foci in the nose. We find that cases which are bacteriologically positive in the nose do as a rule show much more advanced deformities than the negative ones.

Ulcers and deformities - those most deplorable consequences of infection with mycobacterium leprae-can be prevented and can be treated, if their pathogenesis is realised and further studied. Surely there is no lack of patience among those who work with lepers. May part of this patience be diverted to watchful prevention and treatment of ulcers and deformities.

We want to thank the Chiengmai Leper Asylum, which is partly subsidised by the American Mission to Lepers, for supplying facilities and funds for this and other investgations.

\section{DESCRIPTION OF PIHOTOGRAPHS.}

1. Acro-oedema in lepromatous leprosy.

2- 8. Different phases of exercise and band.

9. Padding of ulcers.

I0--1r. Before and after 2 months of our ulcer treatment.

I2. Ulcer treated for 2-3 months. The white line gives the size of the ulcer before treatment.

\section{LITERATURE.}

Klingmueller, Lepra, I930.

Klingmueller, Zentre. hl. f. Haut u. Geschl. kraakh, 57, 5, 32i, 1937.

Rogers-Muir, Leprosy, L928.

Muir, Leprosy, I938.

Cochrane, Leprosy, I929.

Lowe-Chatterji, Lepr. in India, ()ct. I937.

Chatterji, Int. J. Lepr., 5, 3, 1937.

Bosqu., Rev., Ärgent. Dermatosif., ref. Int. J. Lepr., 5, 2, 235.

Grieco, Int. J. Lepr., 6, 3, 36r.

Granger, J. Trop. Med. and Hyg., 40, 1937, I I3.

Haeupl., Muenchner Med. Wschr., 85, 48, 1938.

de Jong, ref. Trop. Dis. I3ull., 3I, 8, 550. 


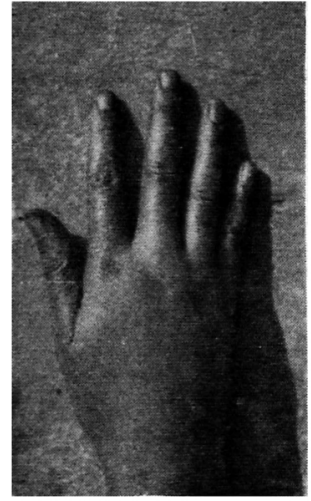

1
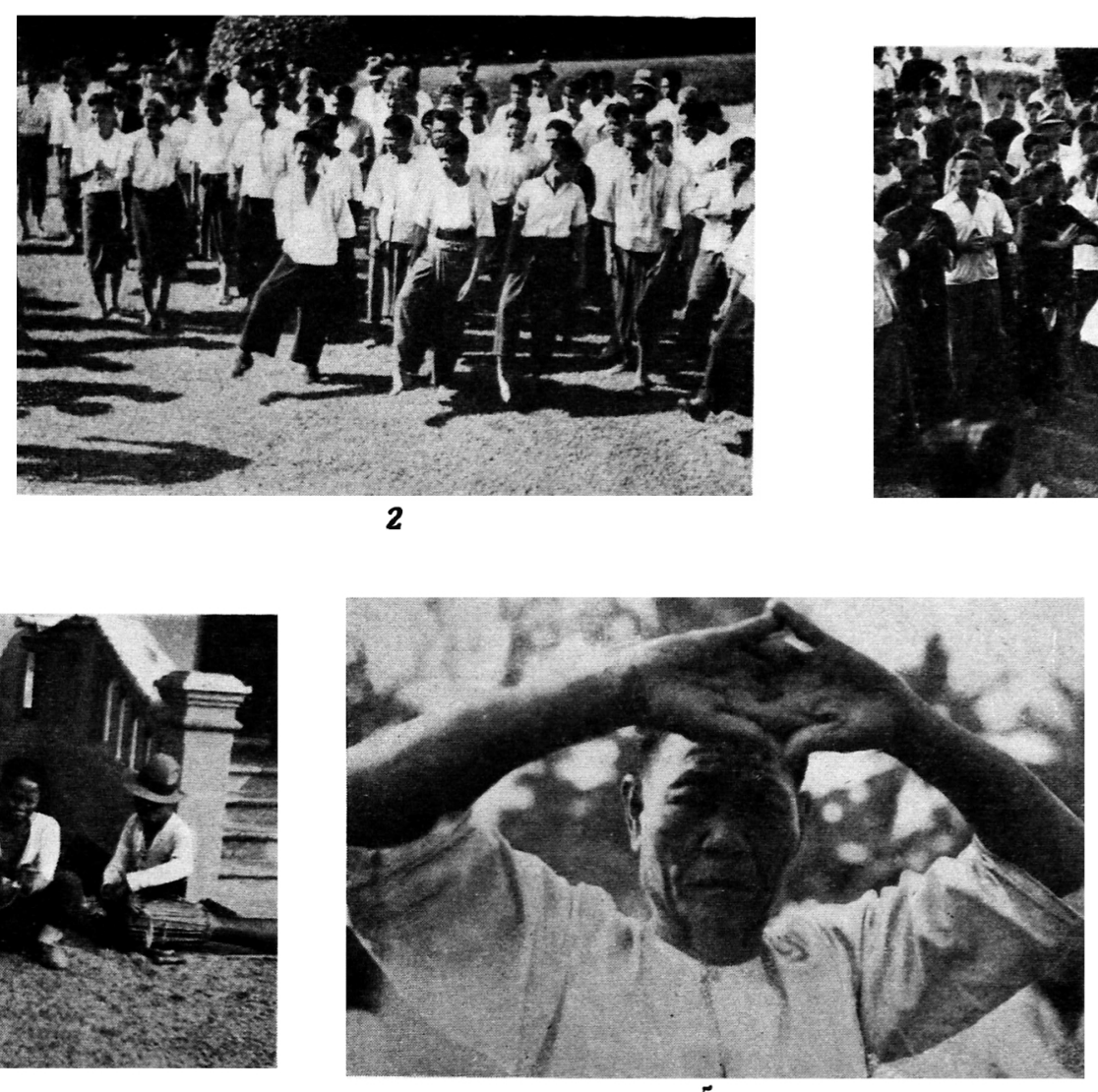

5

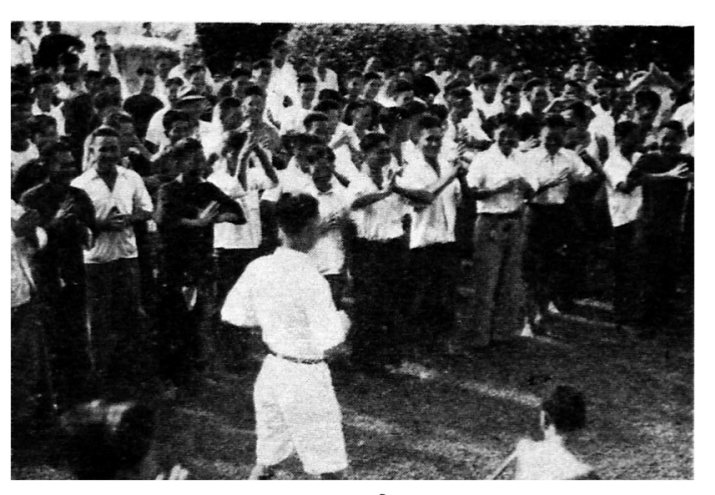

3

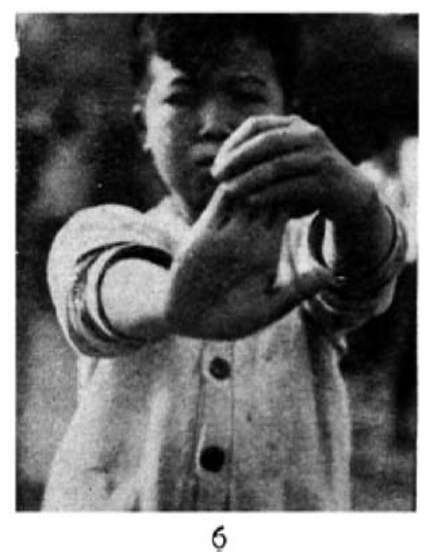



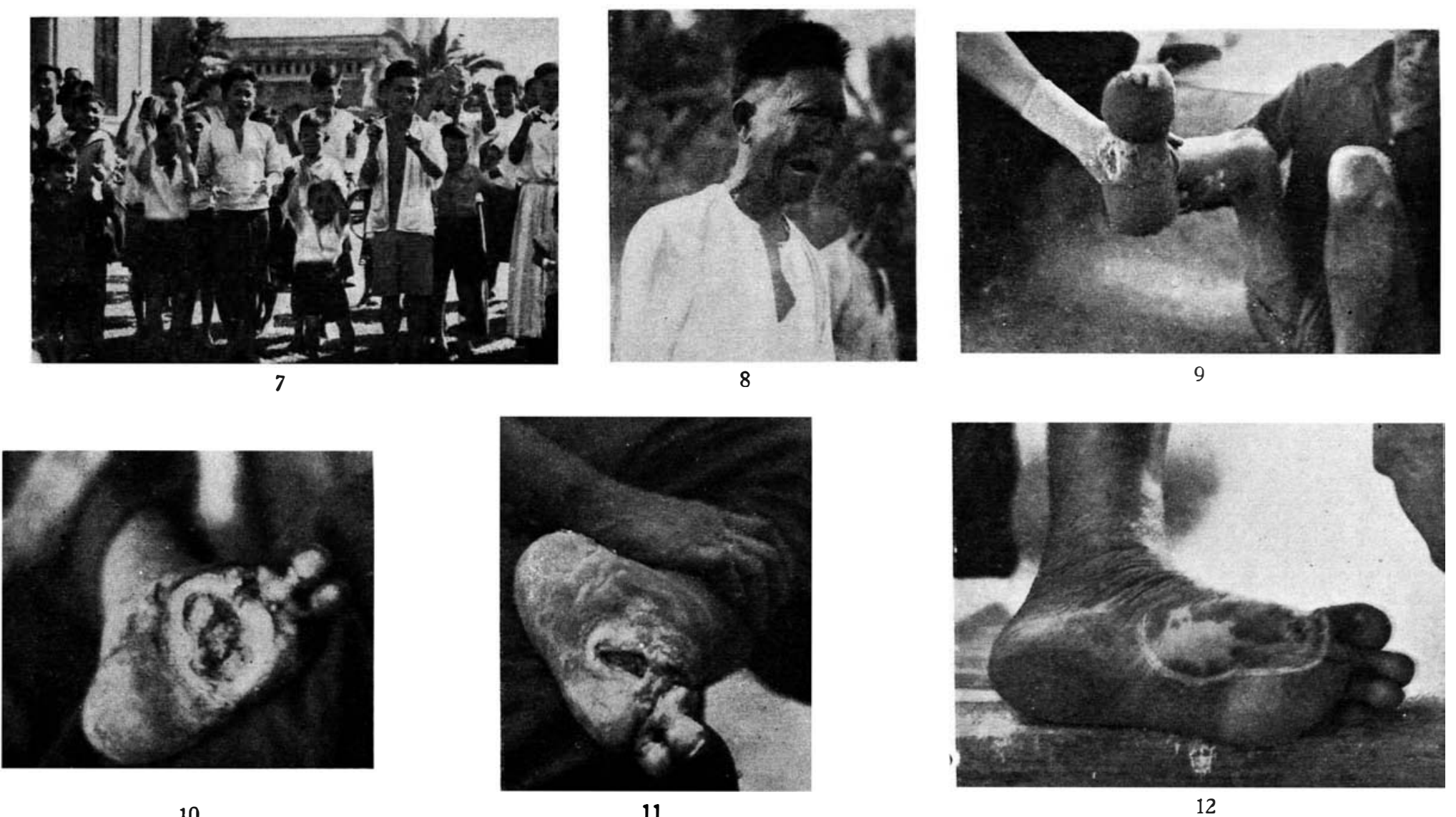
Karaseff, Int. J. Lepr., 5, 3, 360 (ref.).

Ieitner, Int. J. I epr., 6, 4, 471 .

Chopra et: al., ref. Int. J. Lepr., 6, 4, 594.

Cochrane-Raj., Lepr. in India., 9, I937, 18.

Gass., Cairo Congress.

(iupta., Lep. India, 8, I936, 4 I.

(le Moraes, ref. Int. J. Lepr., 5, I, 12 r.

cle la Plaza et al., ref. Trop. 1)is. Bull, 32, I935, $87 \mathrm{I}$.

Dow, Lepr. India, 8, I936, I I3.

Dow-Naragan, Lepr. Review, 6, 2, 1935.

Black, Malayan Med. J., 8, I, 60, I933.

Sakakibara, ref. Int. J. Lepr. 7, 23, 1936.

Ryrie, T., Malayan Br. Brit. Med. Ass., I, 4, 1938.

Bousetield, Int. J. Lepr., 6, I, 73, 1938.

(iass, Iepr. India, 7, 35, I935.

Bet\%, Arch. f. Schiffs u.'Tropenhyg., 42, ro, 468, 1938.

Berny-(ippet, ref. Int. J. Jeppr., 6, 1, r32.

Braga, ref. Int. J. Lepr., 6, I, r 30.

1)ow, leepr. Inclia, 7, I56, 1935.

Floriani, ref. Int. J. Lepr., 6, 1, I32.

(iuida, ref. Int. J. Lepr., 6, I, I32.

Parmakson, Dermat, Wochenschr., 102, r936, I99.

Tisseuil, ref. Int. J. Iepr., 6, 4, 595.

Valente, Int. I. Iepr., 6. 2, 277 (ref.).

Oberdoerffer-Collier, Am. J. Trop. Med., In print. 